Revue des patrimoines

\title{
Un paysage de l'industrie : canaux et usines en Val de Germigny (Cher)
}

Valérie Mauret-Cribellier et Patrick Léon

\section{(2) OpenEdition}

Journals

Édition électronique

URL : http://journals.openedition.org/insitu/8278

DOI : $10.4000 /$ insitu. 8278

ISSN : 1630-7305

Éditeur

Ministère de la culture

\section{Référence électronique}

Valérie Mauret-Cribellier et Patrick Léon, «Un paysage de l'industrie : canaux et usines en Val de Germigny (Cher) », In Situ [En ligne], 6 | 2005, mis en ligne le 19 avril 2012, consulté le 01 mai 2019. URL : http://journals.openedition.org/insitu/8278 ; DOI : 10.4000/insitu.8278

Ce document a été généré automatiquement le 1 mai 2019.

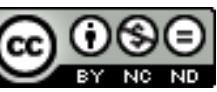

In Situ Revues des patrimoines est mis à disposition selon les termes de la licence Creative Commons Attribution - Pas d'Utilisation Commerciale - Pas de Modification 4.0 International. 


\title{
Un paysage de l'industrie : canaux et usines en Val de Germigny (Cher)
}

\author{
Valérie Mauret-Cribellier et Patrick Léon
}

En forme d'arc de cercle très ouvert, la vallée de Germigny, prolongée au nord par le bassin de Beffes, s'étend sur plus de 50 kilomètres entre Charenton-Laugère (Cher) au sud-ouest et La Chapelle-Montlinard aux portes de La Charité-sur-Loire (Nièvre) au nord (fig. $\mathbf{n}^{\circ} \mathbf{1}$ ). Dominant la Loire et l'Allier, c'est un pays de bois, d'eau et de prairies drainé par l'Aubois et la Vauvise. Sols et climat ont orienté la vallée de Germigny vers l'élevage du charolais au siècle dernier. Aujourd'hui, le tourisme trouve sa place dans l'économie régionale grâce à ses paysages verts, aux châteaux et aux églises romanes. L'industrie est présente avec quelques entreprises implantées avant tout dans les deux petites capitales que sont La Guerche-sur-l'Aubois et Jouet-sur-l'Aubois. Deux usines, la tuilerie Imerys Toiture à Grossouvre et la cimenterie Calcia à Beffes, rappellent l'importance passée de ces deux activités dans une vallée qui connut une industrialisation diversifiée et ancienne. 


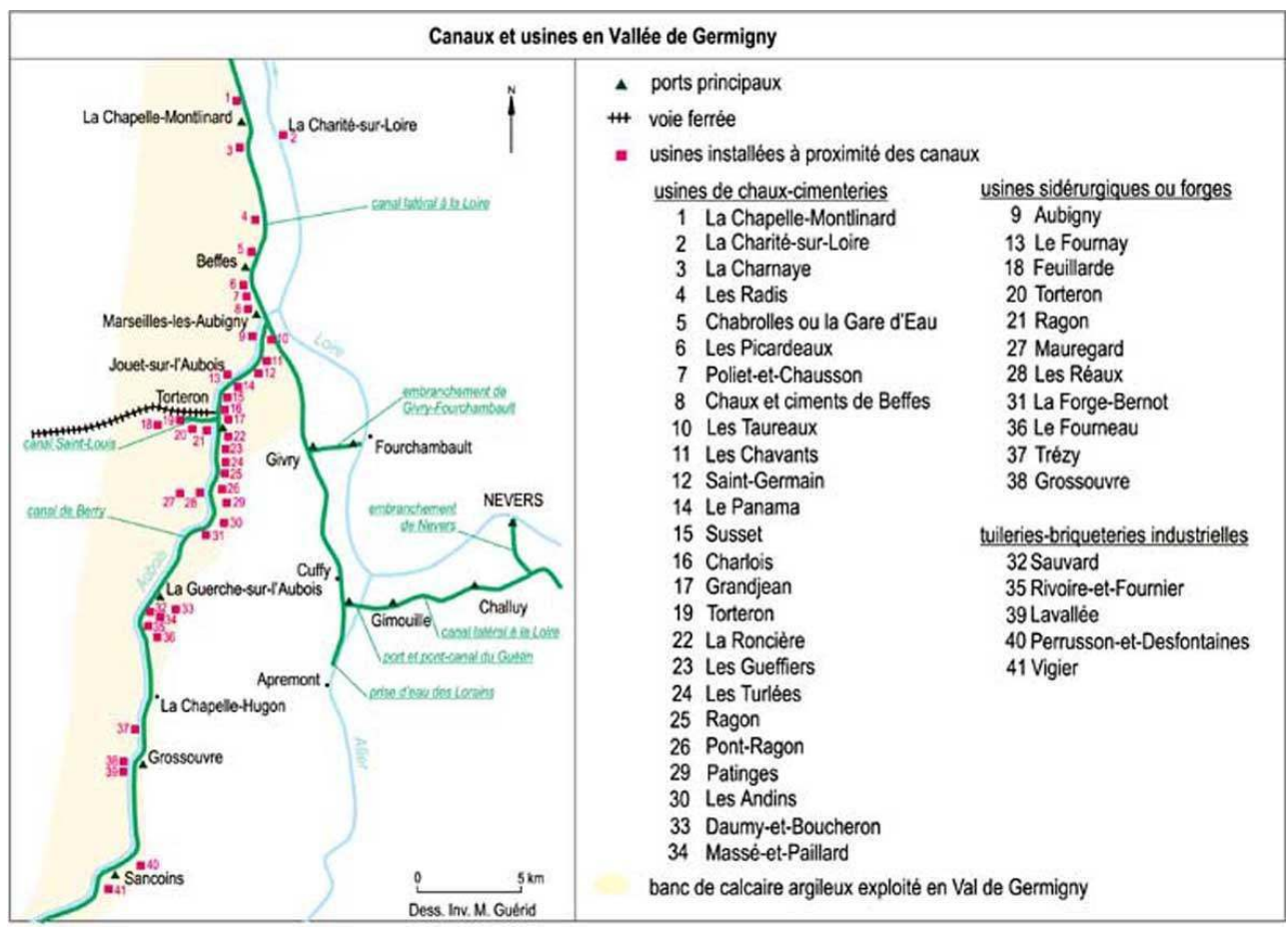

Canaux et usines en Vallée de Germigny

Dess. Inv. M. Guérid @ Inventaire général, ADAGP, 2004

2 L'industrialisation apparaît en effet dès la fin du XIV ${ }^{\mathrm{e}}$ siècle avec la sidérurgie indirecte, à l'instar de plusieurs autres vallées en Berry. La production de fonte et de fer fut la branche dominante jusqu'à son extinction dans les années $1870-1880^{1}$ où le relais est pris par la production en grand de tuile, brique, chaux et ciment qui décline fortement dans les années 1930. Si un paysage façonné par l'industrie existe indéniablement dès les origines, celui-ci demeure ponctuel jusque dans les années 1820-1830. La sidérurgie connaît alors un développement inégalé servi par l'ouverture du canal de Berry et du canal latéral à la Loire qui favorisent par la suite l'installation des activités de la terre dont nous venons de parler.

Entre la construction des canaux dans la décennie 1830 et la grande crise des années 1930, un siècle d'intense activité industrielle a créé un paysage industriel spécifique dont les composantes patrimoniales s'égrènent prioritairement le long des canaux.

\section{I - Des canaux pour la navigation et l'industrie : la formation d'un paysage}

"Ces lignes de navigation verront naître des usines sur leurs bords et dans leur voisinage »: cette phrase prémonitoire - elle date de 1829 - extraite d'un rapport au préfet de l'Allier résume parfaitement notre propos ${ }^{2}$. 


\section{1 - La construction des canaux de Berry et latéral à la Loire ${ }^{3}$, maillons du réseau navigable français, et la sidérurgie (années 1830 - années 1860)}

5 Aux XVII ${ }^{e}$ et XVIII ${ }^{e}$ siècles, le réseau naturel des voies navigables de la France centrale est complété par des canaux essentiellement orientés vers l'approvisionnement de Paris, tels que les canaux de Briare, d'Orléans et du Loing. Ces voies sont élaborées sur le principe d'une Loire navigable. Les conditions difficiles de navigation font que l'on aspire à plus de régularité et de sécurité. Le canal latéral à la Loire et le canal de Digoin à Roanne sont réalisés entre 1822 et 1838 pour suppléer ces insuffisances. Dans le même temps, le canal de Berry relit Marseille-lès-Aubigny et Tours en traversant la province du même nom. Au sein du réseau régional, la zone sud-est composée des canaux de Berry, latéral à la Loire et du Centre est étroitement liée à l'industrie minière 4 et sidérurgique, à l'image du réseau navigable qui se développe à la fin du XIX siècle dans l'Est de la France ${ }^{5}$.

\subsection{Le canal de Berry}

6 Il fut créé pour le commerce et l'industrie des départements de l'Allier, du Cher et de la Nièvre et ardemment souhaité par les sidérurgistes du Berry.

7 Les années 1820-1860 marquent l'apogée des forges en Berry à la fin du premier âge industriel. Ce nouvel ordre sidérurgique passe par une production beaucoup plus importante qui se fait dans des ateliers qui se modernisent. Il faut ajouter le développement de la fonderie, essentiellement à Torteron qui devient l'une des plus grandes fonderies de seconde fusion en France pour la production de matériel roulant de chemin de fer, d'armement et, surtout, de tuyaux pour les canalisations ${ }^{6}$. Les frères Boigues se sont installés sur la rive droite de la Loire, à Fourchambault, où est créée en 1821 une grande forge à l'anglaise grosse consommatrice de houille. Eux puis leurs successeurs ont rapidement pris le contrôle de la dizaine de forges implantées aux XVI et XVII siècles sur l'Aubois lesquelles fournissent de la fonte envoyée dans l'usine mère. Ces usines consomment de grandes quantités de combustible, de plus en plus de charbon « de terre » acheté en dehors du Berry qui n'en a pas, à Decize-La Machine et à Saint-Etienne, charbon qui arrive par les routes et les fleuves avec tous les aléas que ces voies de communication supposent.

8 Il faut donc réaliser une voie navigable - il n'est alors pas question de route- pour amener ce charbon, surtout celui des nouvelles mines de Commentry près de Montluçon plus proches des forges (une centaine de kilomètres), dont les propriétaires cherchent justement des débouchés. Dans l'autre sens, cette voie de communication servira à transporter la production mais aussi le surplus de minerai brut que le Val d'Aubois et les autres minières $d u C^{2}{ }^{7}$, malgré l'intense activité des forges du département ${ }^{8}$, n'absorbent pas. En somme, on espère qu'une voie d'eau artificielle rapprochera minerai, charbon et usines de manière régulière et économique. Il ne peut être question de rendre l'Allier navigable sur tout son parcours, il faut donc un canal. Or la topographie ne s'oppose pas à son tracé qui ne rencontrera pas de forts dénivelés en empruntant les vallées du Cher, de l'Arnon et de l'Aubois.

9 Enfin, les Boigues sont devenus suffisamment puissants pour imposer leurs vues qui rejoignent les préoccupations semblables des autres maitres de forges concernés 
(Charenton, Praud, Bourges, Vierzon, Tronçais). Le canal est ouvert à la navigation en entre 1830 et 1841 , entre 1837 et 1841 en Val d'Aubois'.

\section{2- Le canal latéral à la Loire}

10 Les premiers projets d'un canal latéral à la Loire datent de $1805^{10}$ et sont attribués à Emiliand-Marie Gauthey, concepteur du canal du Centre (1783-1793). L'année suivante, le dossier est confié à l'ingénieur en chef Boistard ${ }^{11}$ qui propose d'établir le canal entre Digoin et Briare, sur la rive gauche de la Loire afin d'éviter les villes et les carrières des coteaux de la rive droite. Malgré l'accord de la Commission des Ponts-et-Chaussées de $1806^{12}$, le Gouvernement ne donne aucun ordre de début d'exécution. On considère que le canal de Berry, commencé en 1807, peut le remplacer en joignant le Bec d'Allier et Tours par l'intérieur des terres et par un tracé plus court. Dans un rapport de 1820 signé par le directeur général des Ponts-et-Chaussées, la ligne Digoin - Tours figure d'ailleurs sous le nom de canal de Berry. Cependant, en dernier lieu, plusieurs compagnies offrent d'exécuter le canal latéral à la Loire et celui-ci est réalisé entre 1822 et 1838.

Le canal latéral à la Loire a été conçu pour pallier les difficultés de la navigation en Loire. Son trafic est étroitement lié lui aussi, aux établissements industriels situés le long de son propre cours, de celui de la Loire et de ceux des canaux voisins. Il apporte aux usines les matières premières (minerai, bois, charbon) et expédie leurs produits finis.

\section{2- Apogée des canaux et second souffle industriel (vers 1860 - milieu des années 1930)}

\section{1- Le trafic et le fret}

Sous l'Ancien Régime, les canaux assurent avant tout une fonction commerciale avec le transport des denrées alimentaires (vins, céréales, poissons, sel, épices) et des matériaux de chauffage (bois, charbon de bois) et de construction (pierre, ardoise), les objets manufacturés et les produits exotiques. Le rôle économique des canaux devient considérable et atteint son apogée entre l'extrême fin du XIX ${ }^{\mathrm{e}}$ siècle et le début du XX siècle $e^{13}$.

Sur le canal de Berry, coke, houille et minerai de fer représentent dès le début une grande part des marchandises transportées. En 1880, ils constituent encore $71 \%$ du fret ${ }^{14}$ sur la ligne Montluçon / Marseille-lès-Aubigny.

En 1855, la houille, le coke et la tourbe forment à eux seuls $75 \%$ du trafic à la descente du canal latéral à la Loire. A la remonte, on compte $50 \%$ de minerai de fer auquel s'ajoutent les fontes et les fers produits par les forges berrichonnes, des matériaux de construction et des produits agricoles ${ }^{15}$. Le minerai de fer transporté par le canal latéral à la Loire provient essentiellement de la région de Dun-sur-Auron (Cher). Il arrive par le canal de Berry et est ensuite dirigé vers des sites métallurgiques, comme Fourchambault ou le Creusot $^{16}$, via le canal du Centre. Le charbon est tiré des mines de Commentry ou de Montceaux-les-Mines puis de celles de Perrecy-les-Forges près de Chalon-sur-Saône et du Nord $^{17}$. 


\section{2- Le cycle de la terre : tuile et brique, chaux et ciment}

15 La sidérurgie s'éteint en Val d'Aubois progressivement dans les années 1860-1870 (Torteron est la dernière usine à fermer, en 1882). La raison principale tient au combustible. L'espoir mis dans les canaux ne se vérifie pas car le transport du charbon est coûteux et interdit de ce fait la production d'acier, aussi les sidérurgistes estiment-ils qu'il vaut mieux installer les usines près des houillères. Les canaux se sont donc retournés contre les intérêts des forges mais ils vont, à l'inverse, remarquablement servir de nouvelles activités industrielles qui s'installent majoritairement sur ses bords, comme les minoteries ${ }^{18}$ et surtout les activités liées à la terre ${ }^{19}$. L'importance de ces dernières se mesure à la nette augmentation du trafic des canaux dans les années 1860-1913.

La production de tuile, brique, chaux et ciment se fait dans de grandes usines mécanisées qui s'implantent pour les premières dans la partie sud, argileuse, du Val de Germigny, et pour les autres au nord, la jonction passant par la petite ville de La Guerche-sur-l'Aubois. Cinq des six tuileries briqueteries de la vallée, qui fabriquent aussi une gamme variée de produits ornementaux, ont choisi la proximité du canal de Berry ${ }^{20}$ et non celle de leur carrière, éloignée de plusieurs kilomètres et souvent desservie par de mauvais chemins ${ }^{21}$. Ce dilemme ne se posait pas pour les usines à chaux et à ciment dont la situation est idéale. En effet, le plateau dominant l'Aubois et la Loire étant un long et profond banc de calcaire, il suffisait de construire les usines entre le canal et le coteau auxquels les fours sont adossés. Sur une largeur de 200 mètres environ en moyenne, se succèdent selon une parfaite logique productive - comme le montre un dessin de l'usine des Radis, commune de Saint-Léger-le-Petit - la carrière, les fours, les halles d'extinction de la chaux vive, de triage (par blutage), de broyage, d'ensachage et de stockage tandis que l'habitat est rejeté aux marges de l'entreprise.

Figure 2

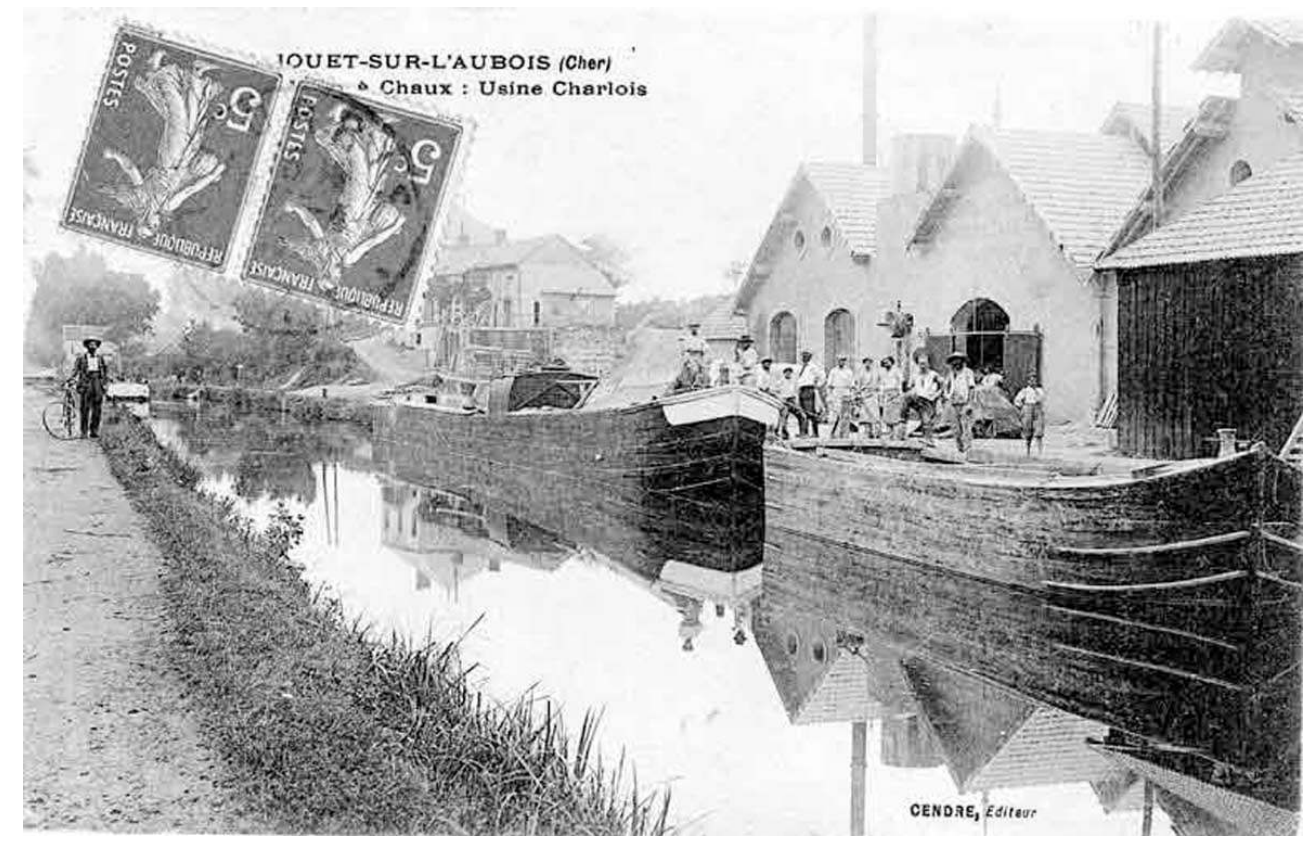

L'usine à chaux hydraulique et ciment naturel Charlois à Jouet-sur-l'Aubois le long du canal de Berry. Carte postale ancienne, Musée Saint-Vic, 18200. Saint-Amand-Montrond

Phot. Inv. R. Malnoury (C) Inventaire général, ADAGP, 1999 
17 Le développement de cette activité est considérable grâce à l'excellent calcaire argileux qui permet de fabriquer de la chaux hydraulique et du ciment naturel particulièrement adaptés à la construction en milieu humide et dont la qualité est garantie par un groupement de producteurs autour de la marque «Beffes». Des 25 usines établies entre La Guerche-sur-l'Aubois et La Chapelle-Montlinard sort $14 \%$ de la production nationale en 1926 (440 000 tonnes) et la vallée de Germigny est alors le second bassin en France après celui du Teil (Ardèche) pour cette production. Avec 1700 employés et ceux occupés dans les activités annexes, la vallée vit au rythme de la chaux et du ciment; la péniche passant une écluse, glissant sur le canal ou arrêtée devant l'usine est, à la veille de la Première Guerre mondiale, l'archétype de la représentation du pays fixée dans les dessins d'amateurs et les cartes postales (fig. $\mathbf{n}^{\circ} \mathbf{2}$ ).

Figure 3

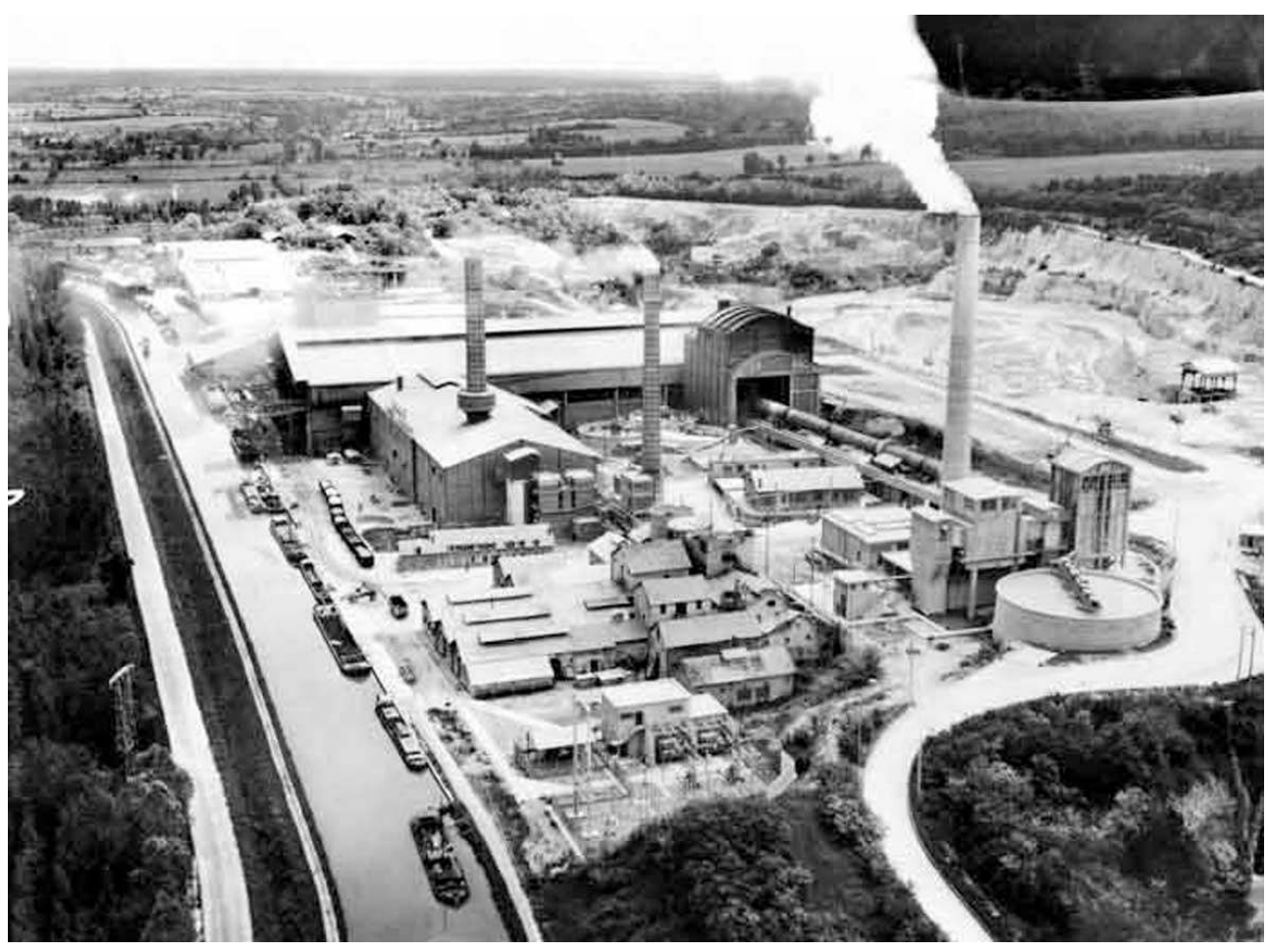

Les établissements Poliet et Chausson (production de ciment artificiel) le long du canal latéral à la Loire, vers 1950. Archives départementales du Cher, fond Lapie

Phot. Inv. R. Malnoury (C) Inventaire général, ADAGP, 1999

Mais ces usines sont trop nombreuses, de taille insuffisante et incapables de prendre le virage du ciment artificiel. Le coup de grâce est donné par la crise des années 1930 qui entraîne leur arrêt, sauf pour les usines que nous avons évoquées : Poliet-et-Chausson (ancêtre de la société Calcia) reconstruite, agrandie et modernisée (fig. $\mathbf{n}^{\circ}$ 3), et la tuilerie-briqueterie de Grossouvre qui s'est spécialisée dans des produits pour la restauration des monuments.

\section{3- le développement des voies ferrées}

19 De 1842 à 1880, plus de 23000 kilomètres de nouvelles lignes de chemin de fer sont ouvertes en France ${ }^{22}$. Les réseaux ferrés et de voies d'eau concourent tous les deux à 
desservir les grands courants commerciaux mais la réalisation des secondes est limitée à certains tracés (problèmes d'alimentation en eau, de mise en œuvre difficile en régions accidentées) et ne peut assurer un maillage aussi fin que les premières. Le chemin de fer, qui présente des avantages incontestables de vitesse et de régularité, longe les canaux car il emprunte lui aussi naturellement les fonds de vallées et dessert lui aussi les usines. Les sidérurgistes et les producteurs de charbon soutiennent le chemin de fer parce qu'il crée un marché: l'usine de Rosières produit des rails, celle de Torteron fabrique des coussinets, le charbon est indispensable au fonctionnement des locomotives...

Figure 4

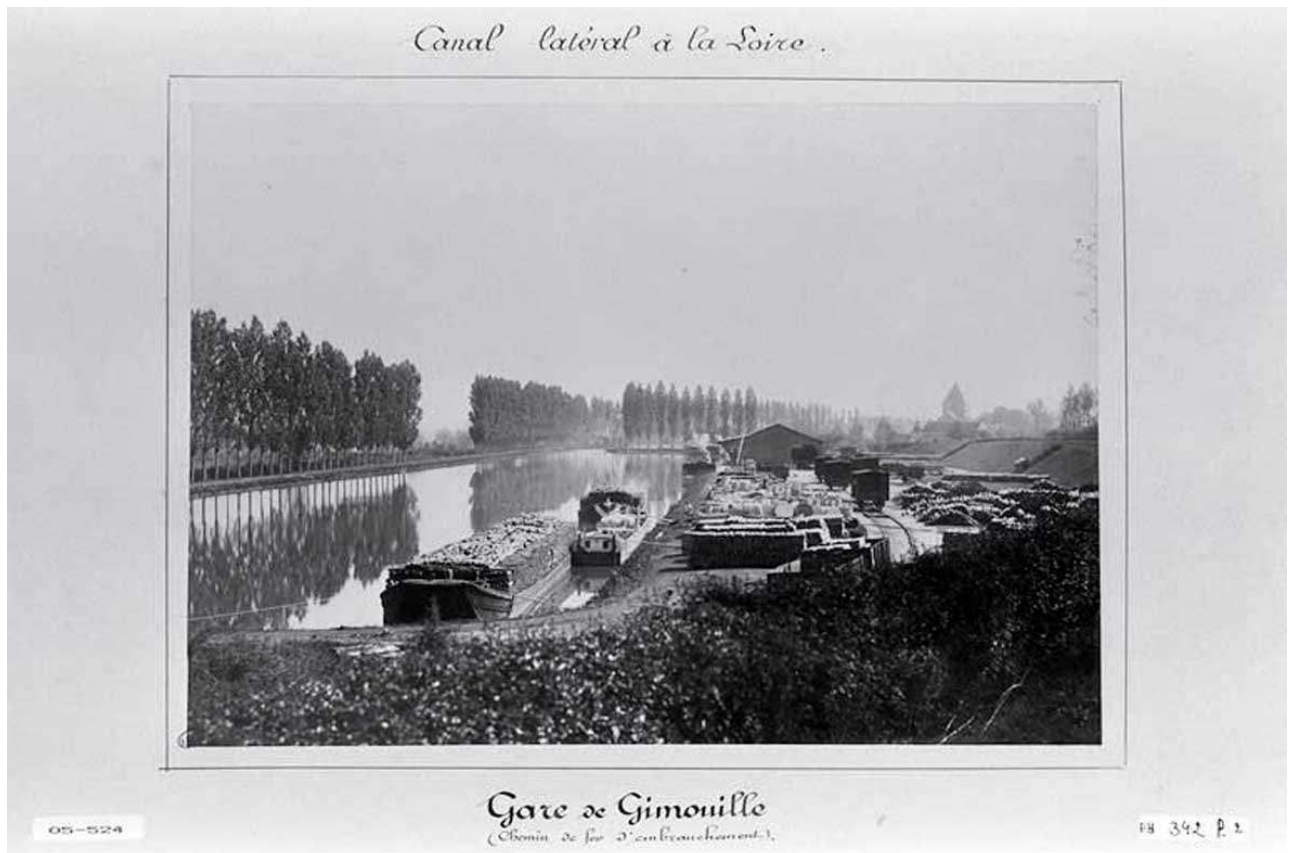

La gare de transbordement de Gimouille à la fin du XIXe siècle

Collection et cliché Ecole nationale des Ponts-et-Chaussées, cote PH 342 P2.

Une concurrence va naître entre les deux systèmes de transports, par le biais notamment de tarifs avantageux ${ }^{23}$. Pourtant, la volonté du gouvernement est de créer une complémentarité entre voie ferrée et canal. Ceci s'exprime notamment dans la liaison de certains ports avec le réseau ferré. A Gimouille (fig. $\mathbf{n}^{\circ} 4$ ), un port de raccordement est relié à la gare de Saincaize (ligne Paris - Lyon par le Bourbonnais) en 1868. 32288 tonnes de marchandises y sont transbordées en $1902^{24}$ dont deux tiers du canal au chemin de fer (houille, chaux hydraulique, ciment, fer, fonte, plâtre, céréales, sable et terre réfractaire) ${ }^{25}$. La construction des gares de raccordement est financée totalement ou en partie par les Compagnies de chemin de fer ${ }^{26}$. Elles reconnaissent ainsi leur intérêt à ces liaisons. Le transbordement s'effectue d'ailleurs essentiellement dans le sens canal - voie ferrée ${ }^{27}$.

\section{II- Patrimoine et paysage de l'industrie en milieu rural}

\section{1 - Une vallée industrielle structurée par les canaux}

Le paysage industriel de la vallée de Germigny se caractérise par la linéarité : il est long d'une trentaine de kilomètres dans la partie que nous étudions mais étroit et fait de 
bandes parallèles : voie ferrée, route, rivière de l'Aubois, canal, usine, carrière, route. A l'intérieur, une zone centrale plus étroite et proprement usinière comprend établissements et canaux, les usines se situant soit à proximité immédiate des canaux soit à une distance de 30 à $50 \mathrm{~m}$. au plus. Au-delà de ces traits communs, la géographie industrielle de la vallée se caractérise par une certaine diversité qui tient à la discontinuité et à la densité. Trois types de situation existent.

Figure 5

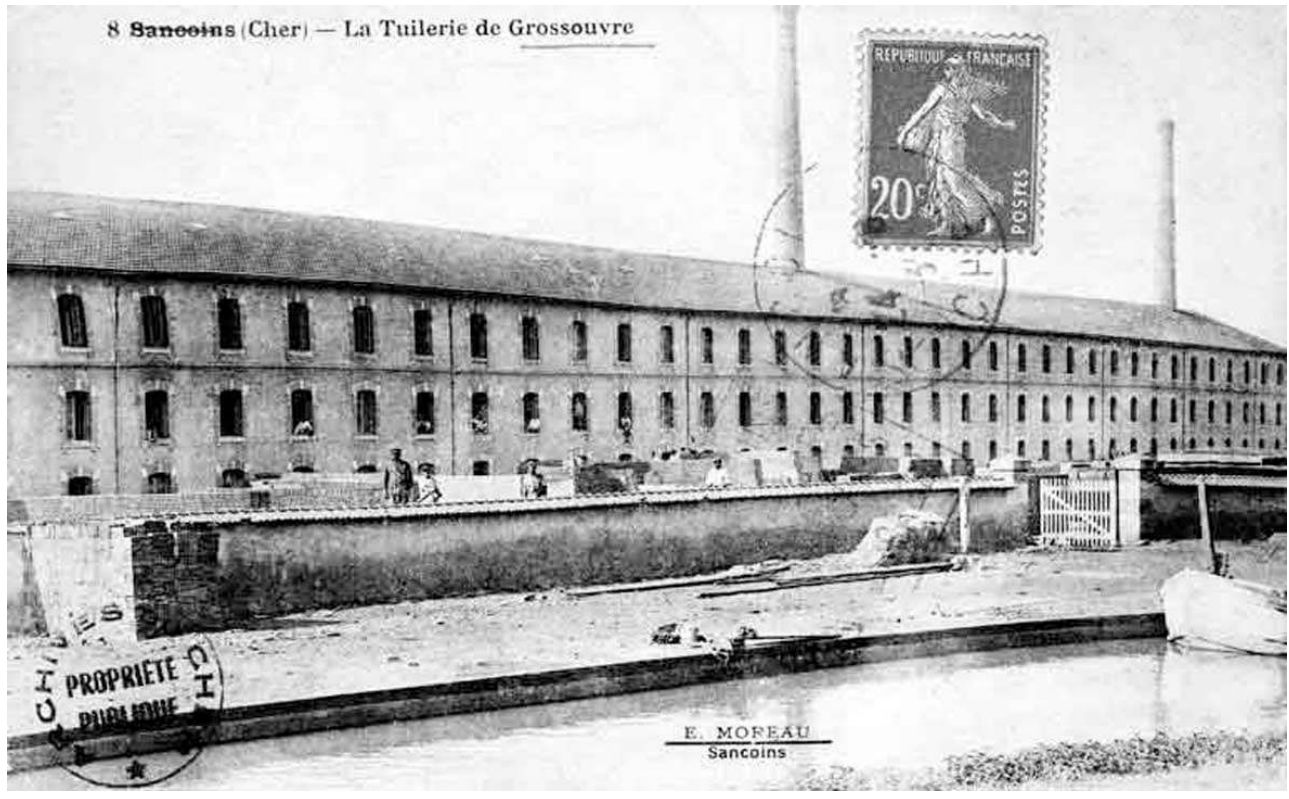

La tuilerie briqueterie de Grossouvre sur le canal de Berry. Carte postale, avant 1914. Musée Saint-Vic de Saint-Amand-Montrond

Phot. Inv. M. Hermanowicz @ Inventaire général, ADAGP, 1992.

$\mathrm{Au}$ sud et au centre de la vallée, l'industrie est présente sous deux formes. En premier lieu, on est frappé par la présence d'usines isolées et vastes, telle la tuilerie-briqueterie de Grossouvre (fig. $\mathbf{n}^{\circ}$ ). Fondée par un banquier de Sancoins, Ernest Lavallée, elle a succédé à une importante forge dont elle a gardé une partie des bâtiments, la halle à charbon et un très novateur immeuble de logements ouvriers dit «Les Galeries ». Elle a aussi beaucoup construit, notamment la halle abritant les fours de type Hoffmann, longue de plus de 200 mètres, comptant trois hauts niveaux, 44 travées sérielles et plusieurs cheminées, surprenant surgissement des symboles de l'industrie en pleine campagne herbagère. En second lieu, on trouve des noyaux plus ou moins compacts en de nombreux endroits: Laugère et Sancoins (tuileries-briqueteries), La Guerche-sur-l'Aubois (sidérurgie, tuile et brique, chaux et ciment, sucrerie et distillerie, moulins et minoteries) pour ne citer que les plus importants. Enfin, dans sa partie nord, de La Guerche jusqu'à La Chapelle-Montlinard, le tissu industriel est très dense. Usines et ports se succèdent en continu au point que dans certaines communes (Beffes, Jouet, Torteron, Marseille-lèsAubigny et Le Chautay dans une moindre mesure), les usines de toute sorte, surtout celles produisant de la chaux et du ciment, se touchent ${ }^{28}$. Les cités ouvrières jouxtant les usines ou insérées dans la trame des villages sont partie intégrante de ce paysage à tel point que, là où elles sont particulièrement nombreuses, elles signalent d'emblée, autant que les usines, l'entrée dans un territoire de l'industrie. 

souvent des usines en hauteur qui dominent le paysage environnant, surtout lorsqu'elles sont représentées du pied de leur carrière qui est l'angle privilégié. On voit d'abord les constructions qui émergent des toits des halles : les hauts fourneaux et les fours à chaux (une douzaine de mètres), systématiquement groupés en longues batteries atteignant 18 fours à l'usine Grandjean de Jouet, les minoteries. L'omniprésence des cheminées crachant de la fumée accentue cette impression. Dernière évidence: l'effet de masse compacte qui émane de ces longs bâtiments accolés ou proches s'étendant parfois sur plusieurs hectares. C'est dans les communes de Beffes, Marseille-lès-Aubigny et Jouet-surl'Aubois que se concentrent les caractères que nous venons de présenter.

\section{2 - Le patrimoine des canaux}

Afin de modérer les dépenses et à la suite d'un voyage d'étude en Angleterre en 1818, l'ingénieur Dutens imagine de réduire les dimensions du canal de Berry. Il s'inspire du réseau navigable anglais qui comprend des canaux principaux à grande section et des petits canaux moitié moins larges dont les petits bateaux «s'attachent " par paire pour emprunter les grandes voies. Les écluses présentent finalement des sas de 2,70 m x 27,75 $\mathrm{m}$. La forme des bateaux a dû être adaptée aux petites dimensions du canal (fig. $\mathbf{n}^{\circ} \mathbf{6}$ ).

Figure 6

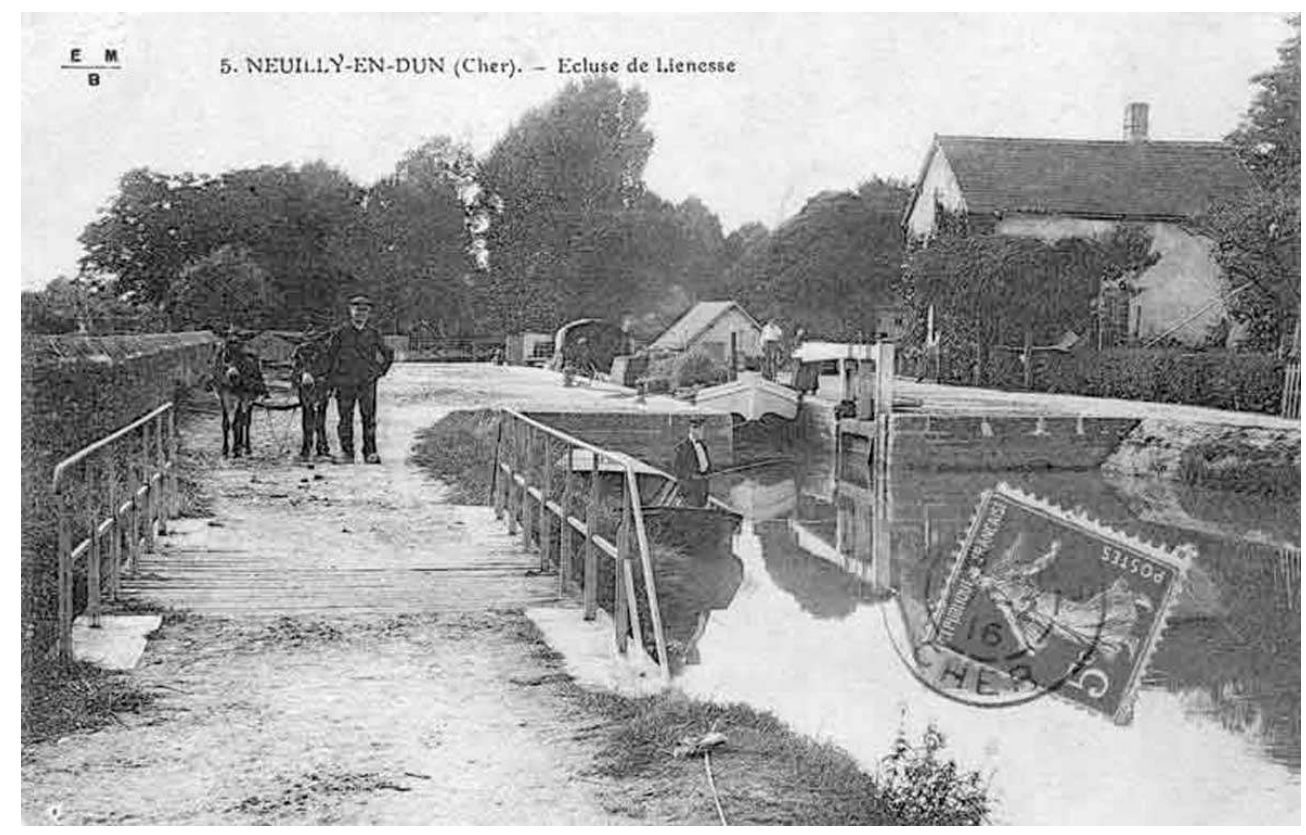

L'écluse de Liénesse à Neuilly-en-Dun sur le canal de Berry (petit gabarit). Carte postale ancienne, Musée Saint-Vic, 18200. Saint-Amand-Montrond

Phot. Inv. R. Malnoury (C) Inventaire général, ADAGP, 1999

Le canal latéral, quant à lui, est construit suivant les gabarits établis par la loi Becquey de 1822 qui impose des écluses de 30,40 x 5,20 mètres. En 1879, la loi Freycinet relative à la classification des voies navigables place le canal latéral à la Loire parmi les lignes principales et impose des sas de $38,50 \times 5,20 \mathrm{~m}$ et la réfection de nombreux ouvrages d'art. Le canal est par conséquent profondément modifié durant cette période. Ces 
travaux accentuent l'écart de gabarit déjà existant entre le canal de Berry non modernisé et le canal latéral.

Figure 7

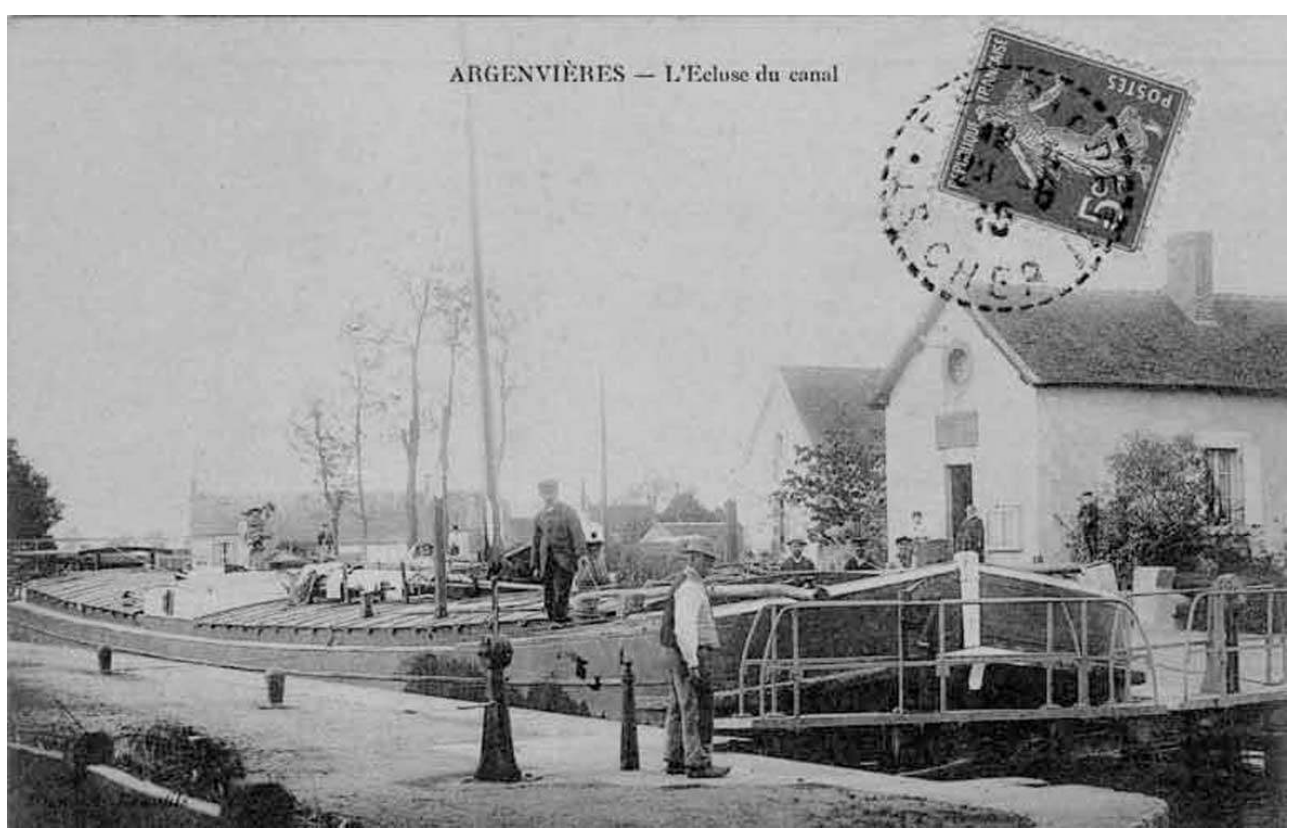

L'écluse et la maison éclusière d'Argenvières sur le canal latéral à la Loire (gabarit Freycinet). Carte postale ancienne, Musée de la Batellerie à Conflans-Sainte-Honorine

Phot. Inv. M. Hermanowicz @ Inventaire général, ADAGP, 1999.

des raisons d'économie, les maisons éclusières du canal de Berry et du canal latéral à la Loire sont construites suivant un modèle simple, propre à chacun des deux canaux (fig. $\mathbf{n}^{\circ} 7$ ). Leur superficie au sol couvre seulement $45 \mathrm{~m}^{2}$ et $50 \mathrm{~m}^{2}$, ce qui correspond aux dimensions habituelles des unités de logement ouvrier de l'époque. Sur le canal latéral, certaines maisons ${ }^{29}$, situées sur des sites «stratégiques", sont beaucoup plus monumentales et présentent des caractéristiques néoclassiques affectionnées par les industriels des années 1830-1840. Généralement, ces maisons abritent, en plus de l'éclusier, des bureaux d'ingénieurs ou de conducteurs de travaux. Parfois, la maison éclusière est au centre d'une véritable mise en scène comme sur le site de la prise d'eau dans l'Allier, aux Lorrains à Apremont-sur-Allier. Les fenêtres de la maison ouvrent en effet sur une écluse circulaire traitée comme un bassin d'agrément et prolongée par une rigole maçonnée et bordée d'arbres.

Certains lieux conservent le souvenir de l'activité engendrée par les voies d'eau. Au Moulin Prunier (Jouet-sur-l'Aubois), la maison éclusière et le bureau de déclaration et de perception côtoient des anciens commerces dont les enseignes peintes encore lisibles indiquent la vente de fournitures (cordes, bâches, articles de marine, feutre, tôle, mercerie), de nourriture pour les ânes tirant les péniches (foin, son, avoine) et de denrées diverses (épicerie, vin en gros, débit de tabac).

En raison de sa linéarité, un canal coupe de nombreuses communications terrestres ainsi que des ruisseaux et des rivières. Les liaisons interrompues sont rétablies au moyen de ponts, d'aqueducs et de ponts-canaux. Près de Nevers, le pont-canal en maçonnerie du 
Guétin (1828-1837) est l'ouvrage le plus remarquable avec ses 18 arches et sa longueur totale de 470 mètres.

\section{3 - La relation entre l'usine et le canal}

\section{1- L'accès direct : l'usine près du canal}

C'est le cas le cas le plus simple bien illustré à nouveau par l'usine à chaux et à ciment des Radis (fig. $\mathbf{n}^{\circ}$ 8). Les halles de stockage ouvrent immédiatement sur le canal, il suffit de traverser le chemin public pour charger ou décharger ; les abords du canal ont été, et à cet endroit seulement, maçonnés pour créer un embryon de quai. Si, lors de la construction des usines à chaux et à ciment, la manutention se faisait essentiellement à dos d'homme ou avec des brouettes ${ }^{30}$ et des charrettes, l'habitude fut vite prise de créer des petites voies ferrées privées pour accélérer les transbordements ${ }^{31}$. Des petits wagonnets à benne basculante, comme on peut encore en voir à l'usine Grandjean à Jouetsur-l'Aubois, transportent les sacs de chaux et de ciment vers les péniches ou le charbon vers les fours. Peu à peu, ces petites voies ferrées irriguent complètement l'établissement qu'elles enserrent (usine Grandjean par exemple ${ }^{32}$ ). On s'en sert aussi à l'intérieur des bâtiments ${ }^{33}$ et elles deviennent indispensables pour acheminer la terre de la carrière à l'usine $^{34}$. On observera qu'elles traversent la voie publique sans que cela provoque apparemment la moindre gêne. Exceptionnellement, ce n'est pas la voie ferrée qui pénètre dans l'usine mais un canal, court et étroit, accessible à une seule péniche qui est dans l'impossibilité de tourner. A la tuilerie Sauvard à La Guerche-sur-l'Aubois un tel embranchement, long d'une centaine de mètres, pénètre dans un espace de stockage clos de murs situé entre canal et rue, devant l'usine.

Figure 8

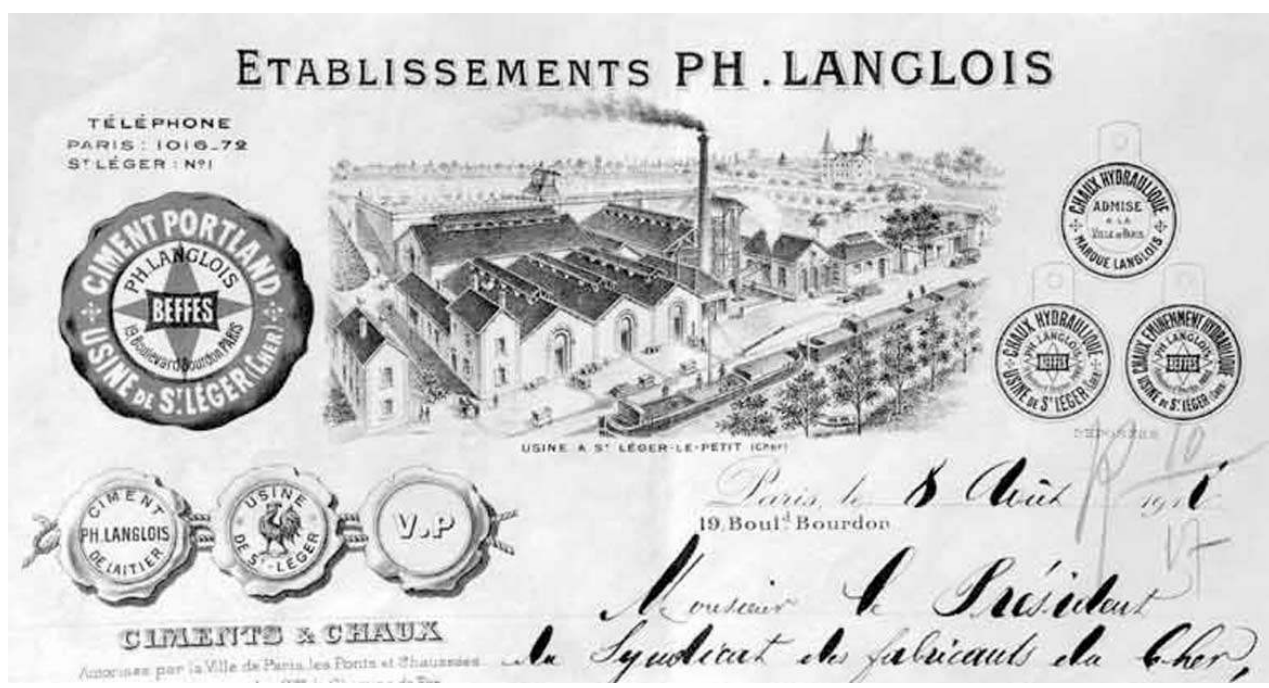

L'usine à chaux hydraulique et ciment naturel des Radis, commune de Saint-Léger-le-Petit près du canal latéral à la Loire. Lettre à en-tête

Phot. Inv. M. Hermanowicz @ Inventaire général, ADAGP, 1991

Certaines usines disposent devant le canal d'un espace clos de murs où les produits finis s'entassent à l'air libre dans l'attente d'être embarqués car ils n'ont pas besoin d'être abrités. La clôture, destinée évidemment à éviter le vol, est percée de plusieurs portes. 
Cette disposition se rencontre dans la plupart des tuileries briqueteries et, parfois, dans les usines à chaux et à ciment.

\section{2- Les embranchements}

Nombre d'usines, notamment les forges, utilisent le canal tout en n'en étant pas proches, il faut alors assurer une liaison. La plupart se satisfont d'un chemin ouvert à leurs frais, comme la « ligne des bœufs » unissant le canal de Berry et le haut fourneau et fonderie de La Guerche-sur-l'Aubois distants de 800 mètres. Mais la connexion par voie d'eau s'est imposée pour les deux plus importantes usines de la vallée, Fourchambault et Torteron, distantes du canal respectivement de 2,4 et 1 kilomètre ${ }^{35}$. Là, l'embranchement s'est imposé. Le canal latéral à la Loire, établi sur la rive gauche du fleuve moins peuplée et moins industrielle que la rive droite, a imposé de relier la voie d'eau artificielle à la Loire par le biais de plusieurs embranchements.

Figure 9

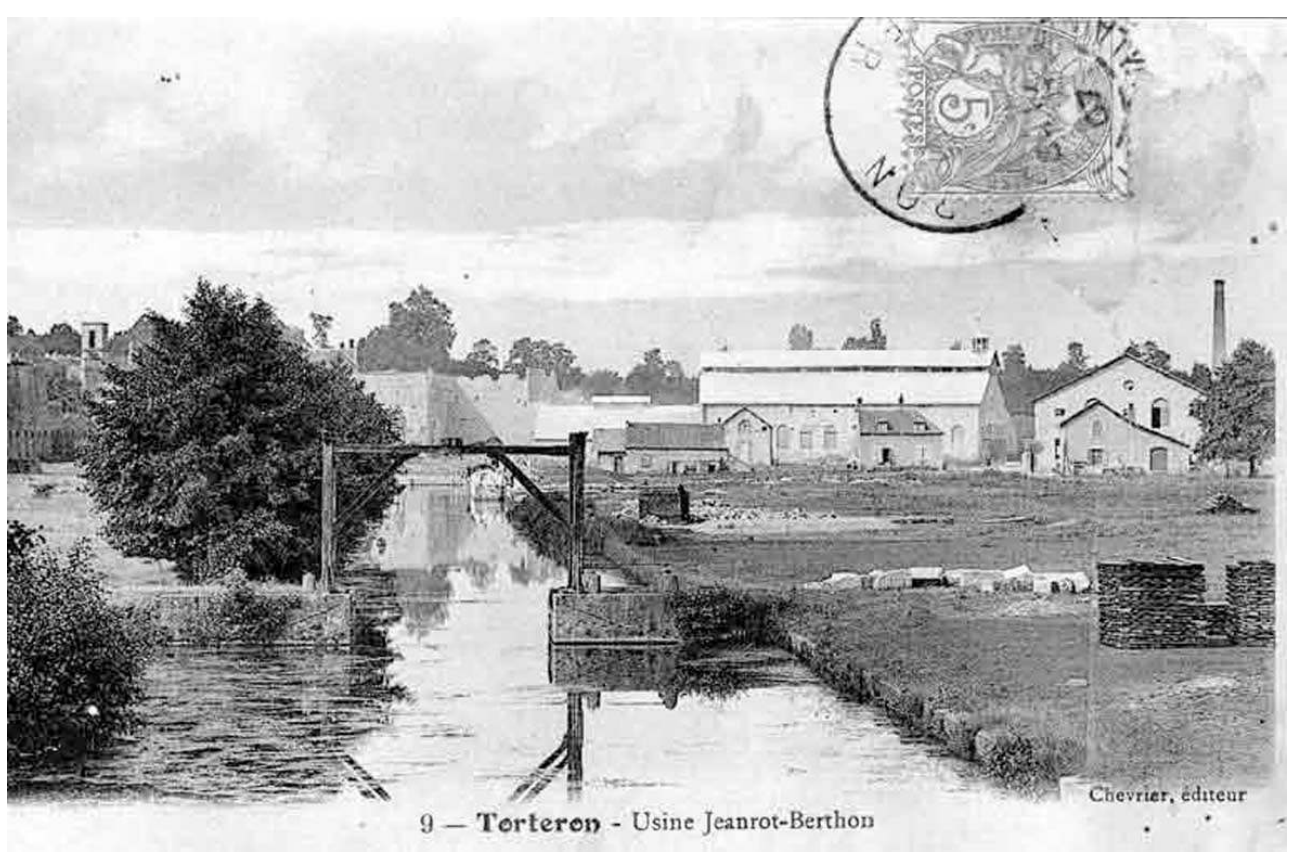

Le canal Saint-Louis dans l'ancienne fonderie de Torteron transformée en usine à chaux hydraulique et ciment naturel. Carte postale ancienne, Musée Saint-Vic, 18200. Saint-Amand-Montrond

Phot. Inv. R. Malnoury ( ) Inventaire général, ADAGP, 1999

Le canal Saint-Louis -du nom de l'un des fils de Pierre Boigues, fondateur de l'empire sidérurgique sur les deux rives de la Loire- a été construit pour relier la fonderie de Torteron au canal de Berry (fig. $\mathbf{n}^{\circ} \mathbf{9}$ ). Long de 800 mètres environ, il fut réalisé entre 1834 et 1838 et opérationnel au plus tard en $1840^{36}$; son ouverture suit donc de peu celle du canal de Berry. Nouvelle manifestation de la puissance des sidérurgistes, son coût a été presque intégralement pris en charge par l'Etat ${ }^{37}$. Sa largeur varie entre $8 \mathrm{~m} 50$ et $15 \mathrm{~m}$ et son gabarit est le même que celui du canal latéral à la Loire dans l'espoir que le canal de Berry soit un jour élargi ${ }^{38}$. Il aboutit au cœur de l'usine, au pied des hauts fourneaux, et un port fut momentanément installé au milieu de l'établissement. Il a fallu franchir l'Aubois par un pont-canal sur lequel nous reviendrons. 


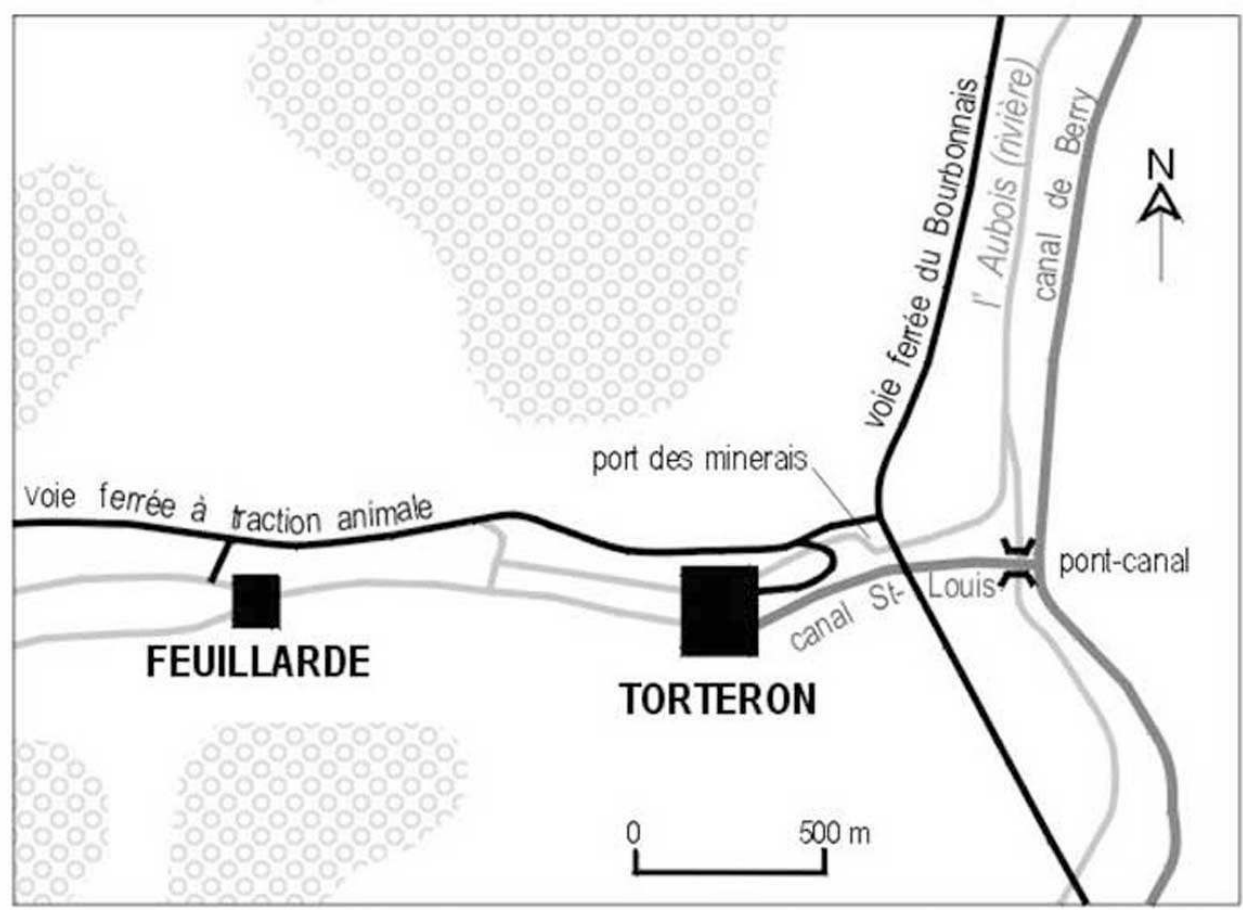

Les voies de communication unissant les usines de Feuillarde-Torteron, le canal de Berry et la voie ferrée du Bourbonnais (années 1860). Sources documentaires : Plan d'ensemble des propriétés Boigues-Rambourg et Cie, 1867 (A. D. Cher 8S12), carte d'Etat-Major et enquête de terrain

Dess. Inv. M. Guérid @ Inventaire général, ADAGP, 2004

La voie ferrée dite du Bourbonnais ouverte en 1861 dans sa partie traversant la vallée de Germigny $^{39}$ détrône petit à petit le canal de Berry (fig. $\mathbf{n}^{\circ} \mathbf{1 0}$ ). Le train est bien plus rapide que la péniche et le chemin de fer passe sur la rive gauche de l'Aubois, là où sont les usines, aussi le trajet qu'exige le transbordement est-il réduit de moitié. Un «port des minerais »-on notera la persistance du vocabulaire lié à l'eau- est installé à la jonction entre cette voie ferrée et le chemin de fer métrique construit par l'usine de Torteron pour amener le minerai des lieux d'exploitation distants de sept kilomètres environ à l'ouest ${ }^{40}$ et prolongé entre cette usine et le port. Cet embarcadère était un espace clos, certes vaste mais sommairement aménagé.

La ligne du Bourbonnais s'avère utile à beaucoup d'usines de la vallée mais elle ne favorise pas, à l'inverse des canaux, l'implantation de nouveaux établissements sur son parcours. Seule, la dernière usine à chaux et à ciment créée dans ce pays, celle des Andins (commune du Chautay) en 1925, en bénéficiera tout en ne renonçant pas au canal. Elle est située entre les deux et on y a construit deux quais maçonnés, singularité parmi ce type d'usines.

L'embranchement de Givry Fourchambault, creusé de 1841 à $1846^{41}$ et long de 2,4 kilomètres, permettait aux bateaux du canal latéral à la Loire d'accéder à la forge de Fourchambault établie en 1821 par Pierre Boigues. Une écluse d'entrée en Loire et des épis établis dans le lit de la Loire facilitaient la traversée du fleuve.

La proximité du canal latéral et les recherches d'Emile Martin, responsable de la fonderie et des ateliers de construction à partir de 1824, ont permis à l'établissement de Fourchambault de remporter de nombreux marchés nationaux et au-delà : localement, 
une passerelle courbe à Briare en 1829 et un pont-canal sur la Vouzance en 1835, des tuyaux de fonte pour des aqueducs en $1839^{42}$, la grue du port de Laugère à Charenton-duCher en 1880, les ornements du pont-canal de Briare en 1890-1896, des ponts métalliques, des portes d'écluses ${ }^{43}$.

Les premières recherches françaises concernant les ponts-canaux métalliques semblent naître au sein des usines de Fourchambault. La connaissance de la fonte et le souci constant de perfectionnement d'Emile Martin, le poussent à de nouvelles expériences. En 1830, le directeur général des Ponts-et-Chaussées et des Mines, J. Berarel, adopte « à titre d'essai ..., la bâche en fonte proposée » et dessinée par Emile Martin pour la traversée de la Vouzance à Molinet (Allier) ${ }^{44}$. Ce pont-canal est réalisé par la fonderie de Fourchambault ${ }^{45}$ entre $1832^{46}$ et $1835^{47}$.

En 1832, Emile Martin propose de construire un pont-canal de 165 mètres avec arches et bâche en fonte reposant sur des piles en maçonnerie pour la traversée de la Loire à Digoin par le canal latéral ${ }^{48}$. Non réalisé, ce projet très osé est finalement remplacé par un pontcanal en maçonnerie.

Figure 11

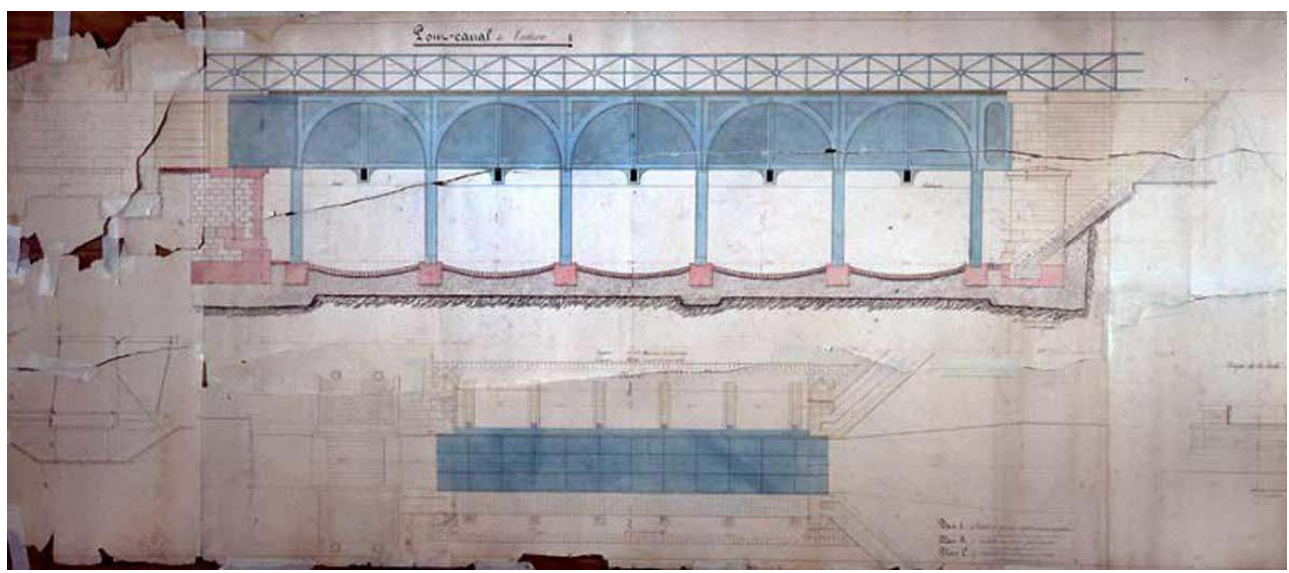

Le pont-canal en fonte sur l'Aubois dessiné le 10 juin 1839, probablement par Greffiths. Document conservé à la DDE de Sancoins

Phot. Inv. M. Hermanowicz @ Inventaire général, ADAGP, 1999

Vers $1839^{49}$, un pont-canal en fonte est construit sur le canal Saint-Louis à Torteron (Cher) dépendant de Fourchambault. L'ouvrage long de 25 mètres enjambait l'Aubois et avait été conçu par un ingénieur mécanicien anglais ${ }^{50}$ travaillant à Fourchambault, George Greffiths ${ }^{51}$. Ce pont-canal disparu est connu par un dessin (fig. $\left.\mathbf{n}^{\circ} \mathbf{1 1}\right)$ et des cartes postales anciennes.

Les datations de ces ouvrages modifient quelque peu la chronologie habituelle des pontscanaux métalliques. Le pont-canal de Barberey-Saint-Sulpice sur le canal de la Haute Seine, considéré comme précurseur en France en 1845-1849, est l'œuvre de Lebasteur, ayant travaillé sur le canal latéral à la Loire entre 1832 et 1839 , sous les ordres de Vigoureux dont il a connaissance des recherches sur les ponts-canaux métalliques. Il s'en inspire lorsqu'il réalise celui de Barberey-Saint-Sulpice. Finalement, les recherches d'Emile Martin, Greffiths et Lebasteur aboutiront à la réalisation par l'ingénieur Mazoyer en 1890-1896 à Briare du plus grand pont-canal métallique jamais construit. 


\section{4 - Les ports}

41 Durant les premières années de service du canal de Berry, l'insuffisance des ports, en nombre et en dimensions, pose de graves difficultés, obligeant parfois les bateaux à parcourir de longs trajets à reculons ${ }^{52}$. Pour faciliter leur stationnement près des usines qu'il longe, la cuvette du canal est parfois élargie : en 1841, à Grossouvre le nombre des bateaux en attente de déchargement ou de chargement s'est élevé jusqu'à 80 et les usagers réclament le creusement d'un port. Pour des raisons identiques, une gare d'eau de 6 x 400 m est creusée près des carrières de pierre à chaux hydraulique de Beffes six ans plus tard ${ }^{53}$.

\section{Figure 12}

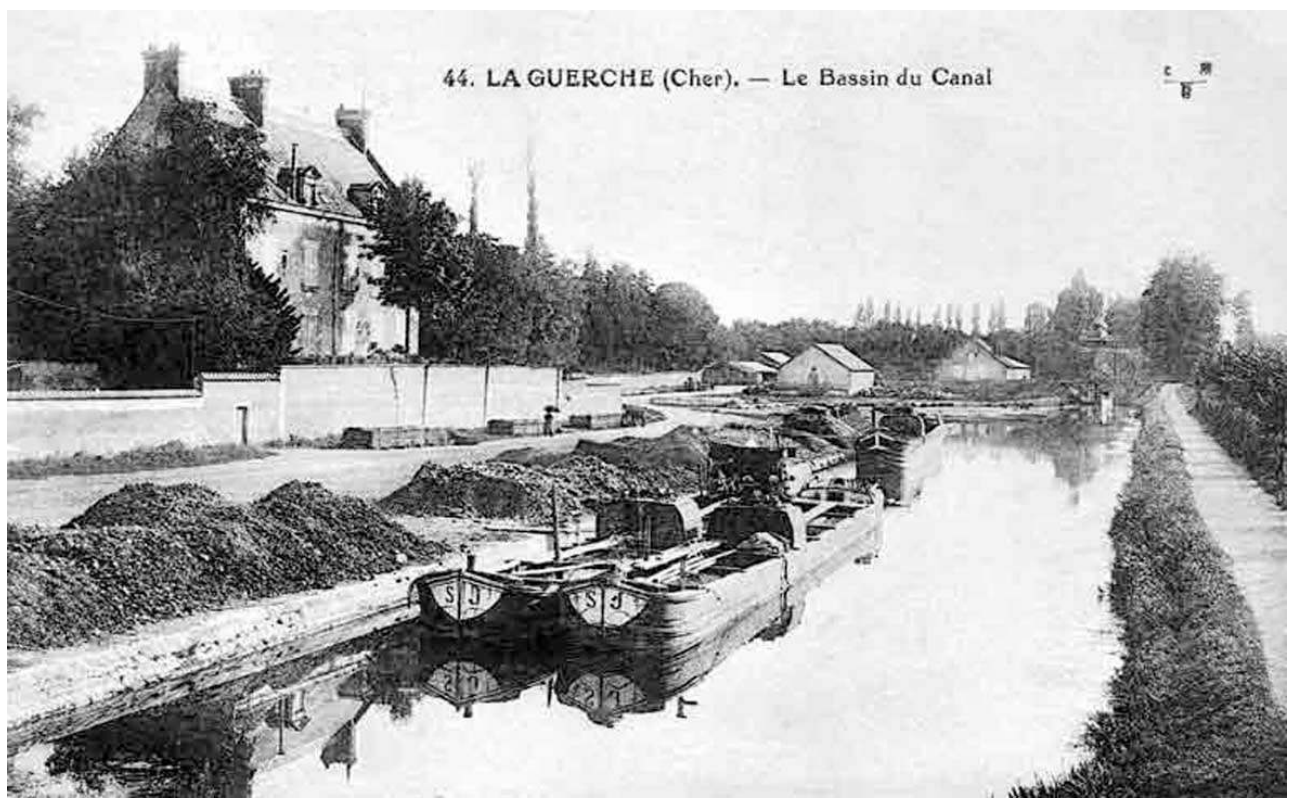

Le port de la Guerche-sur-l'Aubois sur le canal de Berry avant 1914. Le charbon est amoncelé au plus près des péniches; remarquer les péniches allant par deux

Phot. Inv. M. Hermanowicz @ Inventaire général, ADAGP, 1992

Dans la région qui nous intéresse, les principaux ports, étroitement liés aux sites industriels, sont adaptés à la nature des marchandises traitées ${ }^{54}$. Sur le canal de Berry, ce sont ceux de Moulin Pruniers (Jouet-sur-l'Aubois) pour la chaux et le ciment, de la Guerche pour les tuiles et le charbon (fig. $\mathbf{n}^{\circ} \mathbf{1 2}$ ), de Marseille-lès-Aubigny, de Torteron pour les produits métallurgiques, de Dun-sur-Auron pour le minerai, de Sancoins et Grossouvre pour les briques et les tuiles. Sur le canal latéral à la Loire, ce sont les ports de Beffes pour la chaux et le ciment, de Givry - Fourchambault pour les produits métallurgiques et de Marseille-lès-Aubigny ${ }^{55}$. 
Figure 13

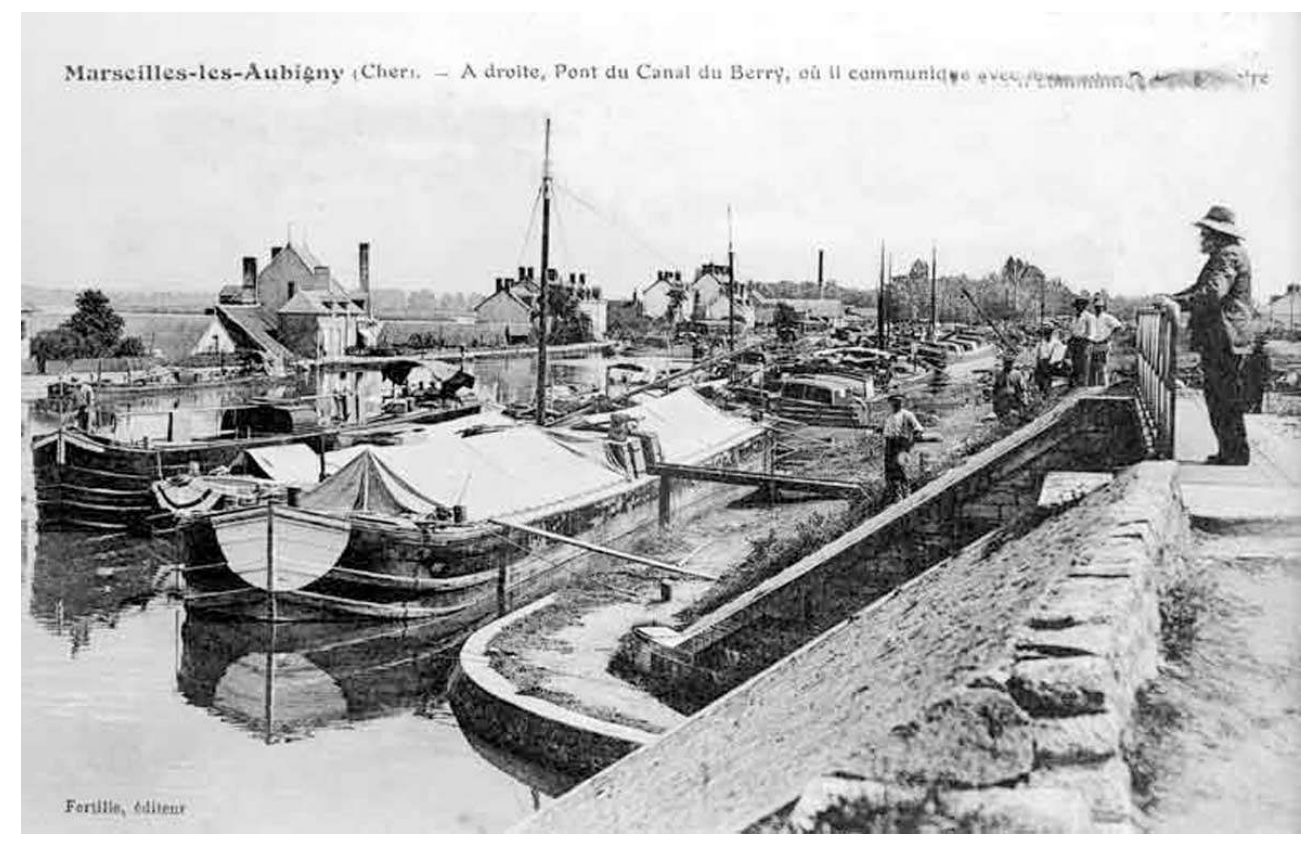

Le port de Marseille-lès-Aubigny, à sa jonction avec le canal de Berry, avant 1914

Phot. Inv. R. Malnoury (c) Inventaire général, ADAGP, 1978

43 Situé au point de rencontre du canal de Berry et du canal latéral, le port de Marseille-lèsAubigny tient une place particulière (fig. $\mathbf{n}^{\circ}$ 13). Il abrite les nombreux transbordements des marchandises entre les grandes péniches du canal latéral et les petites embarcations du canal de Berry (et inversement). En raison de son importance, ce port est agrandi dès le début des années $1850^{56}$. 
Figure 14

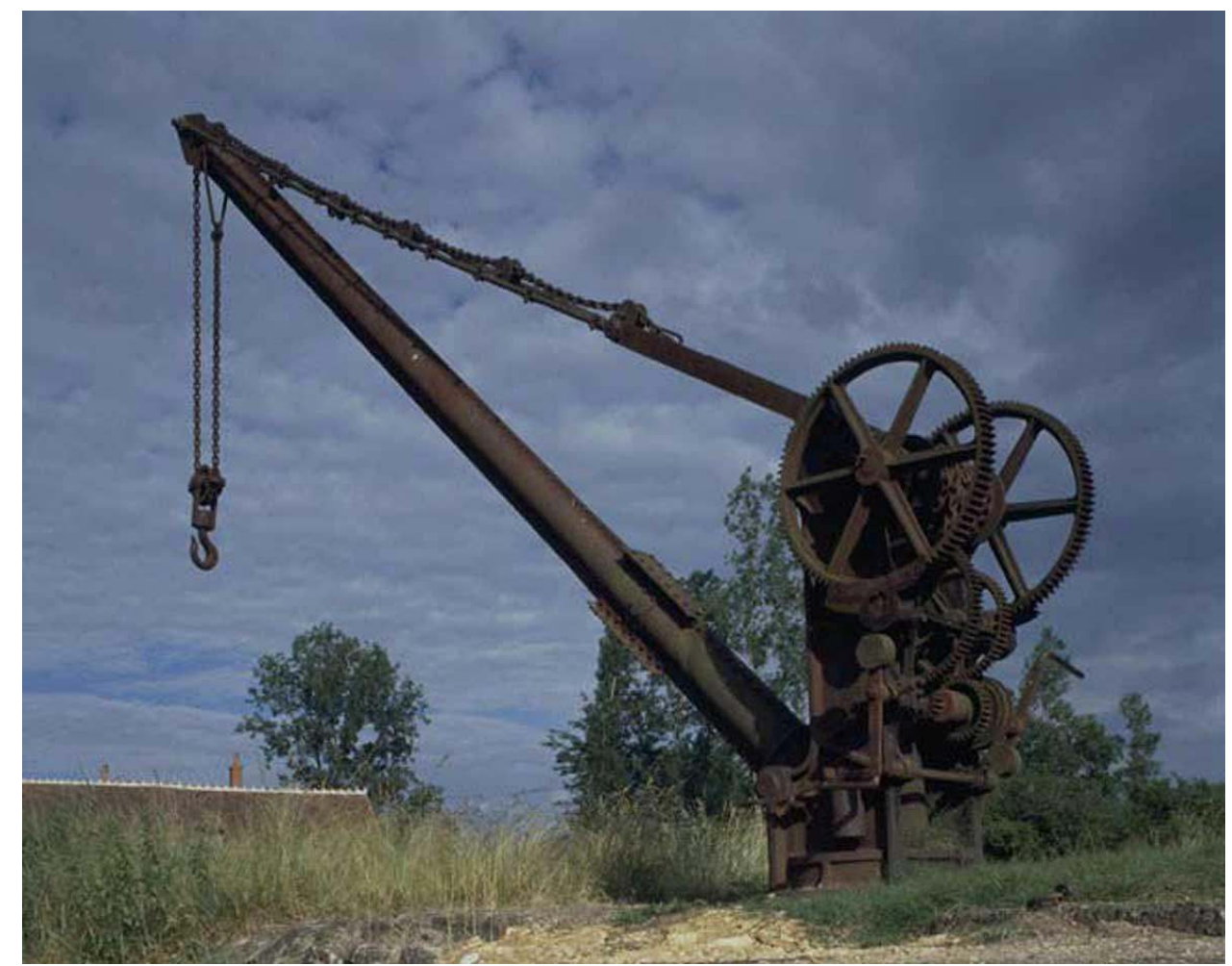

La grue du port de Laugère à Charenton-du-Cher

Phot. Inv. M. Hermanowicz ( ) Inventaire général, ADAGP, 1999

L'équipement des ports est souvent rudimentaire, limité aux quais. Cependant, des installations importantes de manutention existaient dans certains grands ports ${ }^{57}$. Aujourd'hui, seuls deux engins de levage subsistent, aux ports de Gimouille et de Laugère près de Charenton-du-Cher. Ce sont des petites grues à pivot, fixées sur le bord du quai. Celle de Laugère porte la date de 1880 et le nom des fonderies de Fourchambault (fig. $\mathbf{n}$ -14).

Le soin d'outiller les ports publics et d'installer les engins utiles au chargement et au déchargement des marchandises est abandonné à l'initiative des entrepreneurs. Ainsi, la société Boigues-Rambourg organise-t-elle la manutention des marchandises à Montluçon en $1854^{58}$. 
Figure 15

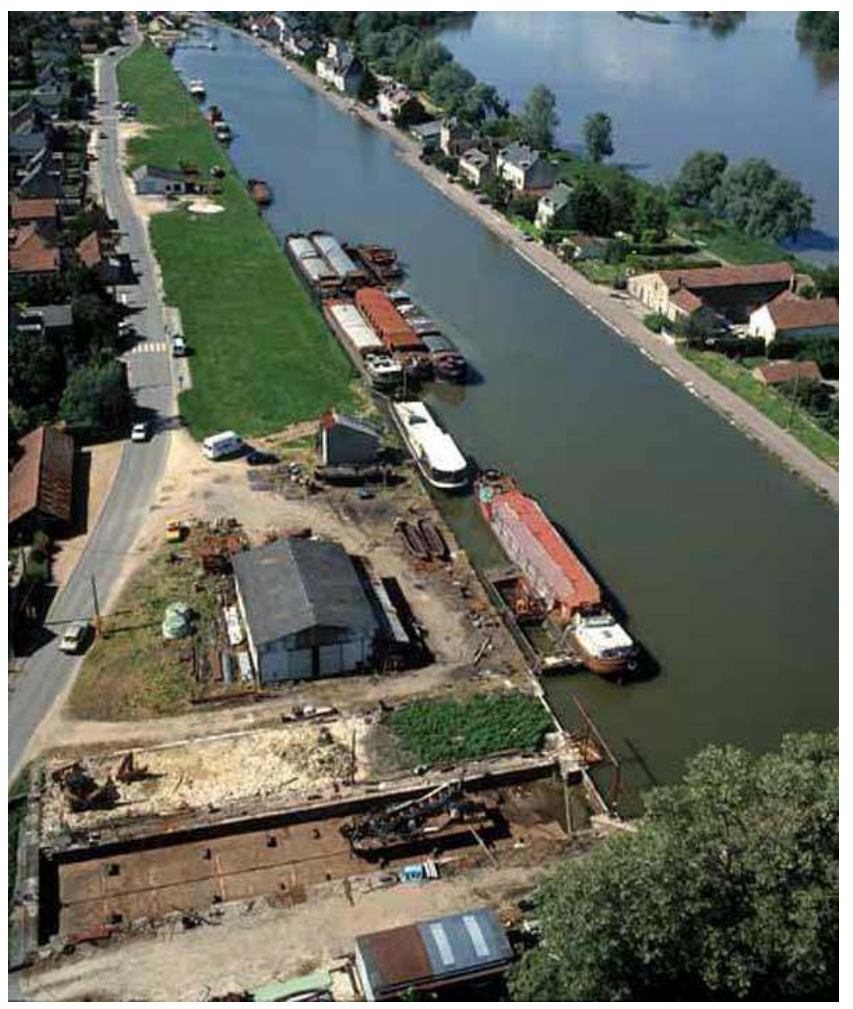

Le port de Marseille-lès-Aubigny vu du sud. Cale de radoub au premier plan

Photographie aérienne de Michel Berger (96 rue des Longues Allées - 45800 Saint-Jean-de-Braye. Tel. 02.38.21.56.77)

Certains ports sont équipés de cales de radoub pour la réparation ${ }^{59}$ souvent situées à proximité de chantiers de construction de bateaux ${ }^{60}$ (fig. $\mathbf{n}^{\circ}{ }^{15}$ ). Vers 1900 , le chantier de Marseille-lès-Aubigny livre à la circulation une moyenne annuelle de 12 bateaux de $30 \mathrm{~m}$ (pour le canal de Berry) et 5 bateaux de 38,50 m (gabarit Freycinet) ${ }^{61}$.

La baisse du trafic du canal de Berry est due à des raisons économiques et commerciales ${ }^{62}$ mais aussi à l'absence de modernisation des ouvrages, à la pénurie d'eau et surtout à l'insuffisance du gabarit du canal. Le déclassement du canal de Berry est prononcé par décret du 3 janvier 1955. Sur le canal latéral à la Loire, on observe également, mais plus tardivement, une forte diminution du transport de marchandises. Après un léger recul pendant la Guerre de 1914-1918, le trafic présente un maximum de 1891000 tonnes de marchandises en 1936. Par la suite, la Deuxième Guerre mondiale porte un coup au développement du canal et le trafic baisse brutalement. Le canal survit tant bien que mal jusqu'aux dernières décennies du XXe siècle, période durant laquelle apparaît le tourisme fluvial qui lui donne une nouvelle activité.

La vallée de Germigny constitue un excellent exemple du développement de la grande industrie en milieu rural, « voie française de l'industrie » définie par Denis Woronoff ${ }^{63}$. De ce paysage industriel il reste des pans entiers ou des vestiges et il est presque toujours palimpseste mais le lien usine/canal est, lui, rarement conservé. Si le canal latéral à la Loire et ses ouvrages sont toujours en place, le canal de Berry se présente de façon tronquée : les portions en eau ou à sec se succèdent et une partie de son trajet a été remblayée. Les chemins de halage de l'un et de l'autre demeurent des lieux de promenade 
agréables. Un projet déjà ancien d'utilisation du canal de Berry pour le tourisme fluvial et patrimonial et pour constituer une réserve d'eau en cas de sécheresse peine à voir le jour.

L'avenir le plus prometteur de ce patrimoine fait d'usines ${ }^{64}$ et de canaux réside dans sa mise en valeur culturelle, économique et touristique par le biais de l'Itinéraire de découverte de l'histoire et du patrimoine de l'industrie du Cher. L'ancienne halle à charbon de la forge de Grossouvre en sera bientôt l'introduction ${ }^{65}$.

\section{BIBLIOGRAPHIE}

\section{Histoire industrielle du Val de Germigny :}

Laurant, Annie. Des fers de Loire à l'acier Martin. Maîtres de forges en Berry et Nivernais. Royer (Saga Sciences). T. 1, 1995 ; T. 2, 1997.

Robin, Raymond. Forges et forgerons du Berry et du Nivernais. Paris : Editions de l'Université et de l'Enseignement Moderne, 1983.

\section{Canaux :}

Flachat, Eugène. Rapport sur le canal de Berry. Paris, 1842.

Mauret-Cribellier, Valérie. Le canal de Berry. Orléans, 2001 (Coll. Itinéraires du Patrimoine, n -239).

Mauret-Cribellier, Valérie. Le canal latéral à la Loire. Orléans, 2004 (Coll. Itinéraires du Patrimoine, $\mathrm{n}^{\circ} 279$ ).

Mazoyer, Léonse-Abel. Des gares de raccordement entre chemins de fer et voies navigables sur les canaux de Roanne à Digoin, latéral à la Loire et du Nivernais. Annales des Ponts-etChaussées, $1^{\mathrm{er}}$ trimestre 1903.

\section{Usines :}

Leon, Patrick. Les forges du Cher. Berry. Une terre à découvrir. 1988, nº 8 ; p. 27-37.

Leon, Patrick. De la connaissance au tourisme industriel : le patrimoine technique de l'usine à chaux et à ciment Grandjean à Jouet (Cher), 1890-1938. Xº colloque du C.I.L.A.C. : Le patrimoine technique de l'industrie, Mulhouse, 19-21 mars 1992. Bulletin de la société industrielle de Mulhouse, $1992, n^{\circ}$ 2, p. 95-102.

Leon, Patrick. Le patrimoine de l'industrie de la chaux et du ciment en Val de Germigny (Cher). Milieu du XIX ${ }^{\mathrm{e}}$ siècle-années 1930. Colloque Fours à chaux en Europe. Documents du Musée de la Pierre, 1996, Fascicule 8, p. 65-88. 
Leon, Patrick et alii. Catalogue de l'exposition Mémoire d'Industries en Val d'Aubois.

Exposition au siège de l'association Aubois de Terres et de Feux ; Le Guétin (Cher), 2002.

\section{Canaux et usines :}

Mauret-Cribellier, Valérie et Leon, Patrick. Un paysage de l'industrie : canaux et usines en Val de Germigny (Cher). L'archéologie industrielle en France. 2004, n 45, p. 39-44.

\section{NOTES}

1. Sur l'histoire de la sidérurgie dans la vallée de Germigny, consulter : Laurant, Annie. Des fers de Loire à l'acier Martin. Maîtres de forges en Berry et Nivernais. Royer (Saga Sciences). T. 1, 1995 ; T. 2, 1997 et Robin, Raymond. Forges et forgerons du Berry et du Nivernais. Paris : Editions de l'Université et de l'Enseignement Moderne, 1983.

2. Citation extraite de Burlin, L. Histoire de l'industrie de la chaux hydraulique et du ciment dans le bassin de Beffes, 1957 ; p. 4 ; étude dactylographiée aimablement communiquée par l'auteur. Voir également les sites suivants. Pour le CILAC (Comité d'information et de liaison pour l'archéologie, l'étude et la mise en valeur du patrimoine industriel) : http://www.cilac.com Pour le TICCIH (Comité international pour la conservation du patrimoine industriel) : http:// www.ticcih.org/.

3. Sur les canaux: Flachat, Eugène. Rapport sur le canal de Berry. Paris, 1842. MauretCribellieR, Valérie. Le canal de Berry. Orléans, 2001 (coll. Itinéraires du Patrimoine, $\mathrm{n}^{\circ} 239$ ). Mauret-Cribellier, Valérie. Le canal latéral à la Loire. Orléans, 2004 (coll. Itinéraires du Patrimoine, $n^{\circ}$ 279). Mazoyer, Léonse-Abel. Des gares de raccordement entre chemins de fer et voies navigables sur les canaux de Roanne à Digoin, latéral à la Loire et du Nivernais. Annales des Ponts-et-Chaussées, $1^{\text {er }}$ trimestre 1903.

4. Déjà au XVIII ${ }^{\mathrm{e}}$ siècle, des projets de canaux résultent de la volonté de désenclaver des lieux de production importants en Berry, notamment les forges royales de Clavières, entre Ardentes et Châteauroux en 1728,1768 et 1772 .

5. Canaux de l'Est, de la Marne à la Saône, de la Meuse à la Saône ...

6. Coulage des tuyaux debout (pour l'adduction d'eau) à partir de 1845. Les principaux marchés furent Paris et Madrid.

7. Les importantes minières de Dun-sur-Auron notamment.

8. Le Berry est un grand pays sidérurgique qui a compté une centaine de forges entre la fin du $\mathrm{XIV}^{\mathrm{e}}$ et le début du XX $\mathrm{XX}^{\mathrm{e}}$ siècle.

9. Grangez, E. Précis historique et statistique des voies navigables en France. 1855, p. 83-94 (chapitre concernant le canal de Berry).

10. Dutens, J-M. Histoire de la navigation intérieure de la France. 1829, t. 1, p 225.

11. Ecole Nationale des Ponts-et-Chaussées : Ms 2733. Rapport du 14 avril 1806 sur le projet de canal de navigation le long de la Loire entre Digoin et Briare par l'ingénieur en chef Boistard.

12. ENPC : Ms 2733. Rapport du 19 mai 1806 par la Commission chargée d'examiner le projet d'un canal de navigation le long de la Loire entre Digoin et Briare par l'ingénieur en chef Boistard. Gauthey est le rapporteur de cette commission.

13. Le trafic du canal latéral à la Loire présente un maximum de 1891000 tonnes de marchandises en 1936, celui du canal de Berry 1600000 en 1896.

14. AN : F ${ }^{14} 6854$.

15. Musée de la Marine de Loire à Châteauneuf-sur-Loire : M 2777. Tableau du mouvement de la navigation dressé par l'ingénieur en chef de Marne. 
16. AN : $\mathrm{F}^{14}$ 6922. Rapport de l'ingénieur Lejeune du 8 novembre 1843.

17. DDE Sancoins : Rapport daté de mars 1911 et concernant les usines de chaux et ciment des environs de Jouet-sur-l'Aubois.

18. Les plus importantes sont celles d'Aubigny, Ragon, Moulin-Prunier, Faguin et Froidefont, vastes bâtiments néo-classiques. On peut aussi citer les nombreux chantiers de construction de bateaux et l'usine de carrosserie hippomobile Rétif, à Sancoins, qui vendait ses carrosses à la reine Victoria et au tsar Nicolas II.

19. Sur les usines de la vallée, voir: Leon, Patrick. Les forges du Cher. Berry. Une terre à découvrir. Hiver 1988, n 8, p. 27-37. Leon, Patrick. De la connaissance au tourisme industriel : le patrimoine technique de l'usine à chaux et à ciment Grandjean à Jouet (Cher), 1890-1938. Xe colloque du C.I.L.A.C.: Le patrimoine technique de l'industrie, Mulhouse 19-21 mars 1992; Bulletin de la société industrielle de Mulhouse, 1992, n 2, p. 95-102, ill., plans. Leon, Patrick. Le patrimoine de l'industrie de la chaux et du ciment en Val de Germigny (Cher). Milieu du XIX siècle-années 1930. Colloque Fours à chaux en Europe. Documents du Musée de la Pierre, 1996, fascicule 8, p. 65-88. LEON, Patrick. et alii. Catalogue de l'exposition Mémoire d'Industries en Val d'Aubois. Exposition au siège de l'association Aubois de Terres et de Feux ; Le Guétin (Cher) ; été-automne 2002.

20. Nous ne parlons pas ici ni des tuileries-briqueteries artisanales ni des petites unités productrices de chaux pour l'agriculture existant dans la vallée dont le marché est surtout local. Certaines d'entre elles ont aussi choisi de s'implanter près des canaux.

21. Sauf la tuilerie-briqueterie de La Rive, commune de Saint-Martin-des-Champs.

22. Alias, J. Naissance et développement du chemin de fer au XIX ${ }^{\mathrm{e}}$ siècle. Annales des Ponts-etChaussées, $n^{\circ} 19,3^{\text {ème }}$ trimestre $1981, n^{\circ}$ spécial, $p 91$.

23. L'avantage du prix reste aux canaux.

24. Mazoyer, L-A. Le Génie civil. 25 novembre 1893, p 50. Voir aussi AD Nièvre : versement DDE 36/1994 SC, dossier 10.

25. AD Nièvre: 3 S 8648.

26. Mazoyer, L-A. Des gares de raccordement entre chemin de fer et voies navigables sur les canaux. Annales des Ponts-et-Chaussées, 1903.

27. AD Nièvre : Niv. 6341. Rapport de l'ingénieur Mazoyer en 1897.

28. Les deux communes voisines de Jouet-sur-l'Aubois et Torteron en totalisent 14.

29. Notamment la maison des Lorrains à Apremont-sur-Allier.

30. Une planche étroite (une dizaine de centimètres) était tendue entre le quai et la péniche.

31. Le fait est attesté à l'usine de la Société Anonyme des Chaux Hydrauliques de Beffes en 1881 ( Album industriel et financier illustré. Paris : A. Chaix, 1881, gravure p. 248) mais on ignore à quelle date ces voies furent installées.

32. Et dans une moindre mesure à l'usine de Chabrolles ou de la Gare d'Eau à Beffes (site restauré ouvert au public).

33. Tuilerie Sauvard à La Guerche, vers 1900, pour charger le four Hoffmann.

34. C'était le cas à la tuilerie-briqueterie de Grossouvre par exemple.

35. D’autres embranchements privés ont été réalisés, tels celui transportant le minerai de fer du bassin de Taissiau près de Dun-sur-Auron et celui desservant les hauts fourneaux de Mazières près de Bourges.

36. Le creusement est en cours en 1834 ( $c f$. premier cadastre). Il est terminé vers 1838 selon Jolant, E. Les usines de Torteron. Histoire de la métallurgie en Berry, Bourges. Les Orphelins du Centre, s.d. (après 1925), p. 37.

37. L'Etat a payé 700000 francs sur un total de 800000 francs (Thuillier, G. Georges Dufaud et les débuts du grand capitalisme dans la métallurgie en Nivernais, au XIX ${ }^{\mathrm{e}}$ siècle. Paris : SEVPEN, 1959 ; p. 77).

38. Grangez, E. Précis historique et statistique des voies navigables en France, 1885, p. 93. 
39. Dancoisne, P. Théorie des graphes et constitution du réseau ferré français. Thèse de géographie, sous la direction de Ph. Pinchemel, Paris I, 1984.

40. La voie ferrée amenant le minerai a été réalisée au milieu des années 1850 en même temps que la modernisation de l'exploitation minière à l'ouest de Torteron (Jolant, E. Les usines de Torteron. Histoire de la métallurgie en Berry, Bourges. Les Orphelins du Centre, s.d. (après 1925), p. 54).

41. A cette date, 1950 bateaux empruntent cette voie d'eau et 34000 tonnes y transitent.

42. AD Loiret : 3 S 8549.

43. Emile Martin a exposé des portes d'écluses en fonte, fer et bois aux salles de l'Industrie, place Louis XV, en 1834. Voir aussi ENPC: Ms 1777.

44. AD Allier: 3 S 2657.

45. AD Allier: 3 S 2657.

46. AD Allier : 3 S 2679. Adjudication des travaux de construction de l'aqueduc de la Vouzance le 25 août 1832.

47. Rapport au roi sur les canaux et autres ouvrages entrepris en vertu des lois des 20 juin et 5 août 1821 et 17 avril et 14 août 1822. Le rapport de mars 1835 rapporte que la bâche en fonte du pont-canal de la Vouzance est terminée. Cet ouvrage est remplacé par une cuvette en maçonnerie à la fin du XIX ${ }^{\mathrm{e}}$ siècle.

48. AN : $\mathrm{F}^{14} 10101 / 3$, pièce 52.

49. DDE Sancoins : élévation et plan d'un pont-canal en fonte sur l'Aubois, 10 juin 1839, non signé mais probablement de Greffiths.

50. En Angleterre, la première cuvette en fonte soutenue par des piles en maçonnerie apparaît en 1795. D'après Marrey, B. Les ponts modernes, $\mathbf{1 8}^{\mathrm{e}}$ et $19^{\mathrm{e}}$ siècles, 1990, p 242.

51. Jolant, E. Les usines de Torteron. Histoire de la métallurgie en Berry, Bourges. Les Orphelins du Centre, s.d. (après 1925), p 39.

52. Flachat, E. Rapport sur le canal de Berry, 1842, p. 89-92.

53. AD Nièvre : versement DDE 47/1996, S/L, dossier 48.

54. AD Nièvre : versement DDE 36/1994 S/C, dossier 10. Texte daté de 1914.

55. AD Nièvre : Niv. 6341. Rapport de l'ingénieur en chef Mazoyer, 1897 et rapport de Mazoyer de 1902.

56. AD Nièvre : versement DDE 47 / 1996, S/L, dossier 48.

57. Guide de la navigation intérieure, 1892, p 19.

58. Besson, A. Notice historique sur les industries à Montluçon, le canal (1808-1955). 1982.

59. Marseille-lès-Aubigny, la Chapelle-Montlinard, Nevers, Sancoins, Dun-sur-Auron, Vierzon, Saint-Amand-Montrond, Montluçon et l'embranchement de Saint-Satur.

60. C'est notamment le cas à Marseille-lès-Aubigny, à Sancoins, à Dun-sur-Auron, et à Montluçon. 61. AD Nièvre : versement DDE 47/1996, S/L, dossier 46.

62. La nette diminution du trafic de la section de Montluçon au début du XX $\mathrm{XX}^{\mathrm{e}}$ siècle correspond aux progrès de la métallurgie en Lorraine et à l'abandon des mines de charbon de Commentry : ce qui avait constitué l'essentiel du fret disparaît peu à peu. Jusqu'à la fin, les usines apportent la plus grosse partie du fret sur la branche de Marseille-lès-Aubigny.

63. Woronoff, D. Histoire de l'industrie en France : du XVI ${ }^{\mathrm{e}}$ siècle à nos jours. Paris : Seuil, 1994.

64. Plus d'une centaine d'usines en place (sans compter les activités artisanales et semiindustrielles), près de 300 fours à chaux conservés, des cités ouvrières construites entre le XVIII siècle et $1951 \ldots$

65. Leon, P., Lecomte, E. Un sens en construction. L'itinéraire de découverte de la Vallée de Germigny (Cher). L'Archéologie industrielle en France, $n^{\circ}$ 40, juin 2002, p. 20-27. 


\section{RÉSUMÉS}

Le canal de Berry et le canal latéral à la Loire, qui se rejoignent au port de Marseille-lès-Aubigny, ont été ouverts à la fin des années 1830 . Ils constituent un maillon essentiel du réseau de canaux dans la France centrale. Le canal de Berry, voulu par les sidérurgistes berrichons pour approvisionner les forges en charbon, a rapidement joué un rôle déterminant dans l'implantation de nouvelles usines entre le milieu du XIX ${ }^{\text {e }}$ siècle et les années 1920, surtout les tuileriesbriqueteries et celles produisant de la chaux hydraulique et du ciment naturel. Pour les mêmes raisons, ces dernières ont aussi choisi les bords du canal latéral à la Loire qui a été initialement conçu pour pallier les insuffisances de la navigation sur le fleuve. Les canaux seront d'abord considérés comme des ouvrages hydrauliques, architecturaux et techniques mais on verra aussi le trafic et le fret qui les animent. Ils sont enfin les serviteurs des grandes usines qui en dépendent étroitement pour leur approvisionnement et l'écoulement de leur production. Par conséquent, un paysage particulier s'est constitué, caractérisé par le lien organique entre canal et usines selon une géographie linéaire : formes de la proximité de l'établissement industriel par rapport à la voie d'eau artificielle, embranchements particuliers (canaux, voies ferrées et ponts), ports.

The Berry canal and the lateral canal running alongside the Loire, which meet up at the port of Marseille-lès-Aubigny, were cut at the end of the 1830s. They are an essential part of the network of canals in central France. The Berry canal, originally created for the iron and steel producers of the Berry region in order to facilitate coal supplies to their ironworks, became a vital influence in the implantation of new factories, from the middle of the nineteenth century up to the 1920s. Of particular importance were the brick and tile works, and other factories producing hydraulic lime and natural cement. Similar factories also sprang up along the canal running beside the Loire, originally designed to avoid the obstacles to navigation on the river itself.

\section{INDEX}

Mots-clés : canal de Berry, canal latéral Loire, forge, tuilerie, navigation fluviale, voie d'eau, Germigny, Cher, Allier, Cimenterie, Sidérurgie, réseau navigable, canal du Centre, industrie minière, Nièvre, Torteron, Fourchambault, Aubois, Commentry, Boignes, Gauthez, Transport, four à chaux, haut fourneau, minoterie, écluse, canal Saint-Louis, pont-canal, Marseille-lesAubigny

Keywords : factories, hydraulic lime, cement

\section{AUTEURS}

\section{VALÉRIE MAURET-CRIBELLIER}

Chercheur. Inventaire. Région Centre. valerie.mauret-cribellier@culture.gouv.fr 


\section{PATRICK LÉON}

Conservateur du patrimoine. Inventaire. Région Centre. patrick.leon@culture.gouv.fr 